\title{
In-plane magnetoconductivity of Si MOSFETs: A quantitative comparison of theory and experiment
}

\author{
S. A. Vitkalov, ${ }^{1}$ K. James, ${ }^{1}$ B. N. Narozhny, ${ }^{2}$ M. P. Sarachik, ${ }^{1}$ and T. M. Klapwijk ${ }^{3}$ \\ ${ }^{1}$ Physics Department, City College of the City University of New York, New York, New York 10031 \\ ${ }^{2}$ Department of Physics, Brookhaven National Laboratory, Upton, New York 11973-5000 \\ ${ }^{3}$ Department of Applied Physics, Delft University of Technology, 2628 CJ Delft, The Netherlands
}

(Received 12 December 2002; published 19 March 2003)

\begin{abstract}
For densities above $n=1.6 \times 10^{11} \mathrm{~cm}^{-2}$ in the strongly interacting system of electrons in two-dimensional silicon inversion layers, excellent agreement between experiment and the theory of Zala, Narozhny, and Aleiner is obtained for the response of the conductivity to a magnetic field applied parallel to the plane of the electrons. The parameters deduced from fits to the magnetoconductance do not provide quantitative agreement with the observed zero-field temperature dependence. We attribute this to the neglect in the theory of additional scattering terms, which affect the temperature dependence more strongly than the field dependence.
\end{abstract}

DOI: 10.1103/PhysRevB.67.113310 PACS number(s): 73.40.Qv, 71.30. +h, 71.10.Ay, 72.10. $-\mathrm{d}$

Two-dimensional (2D) systems of electrons ${ }^{1-3}$ and holes $^{4-8}$ have been the focus of a great deal of attention during the last few years. ${ }^{9}$ Their resistance exhibits a metallic temperature dependence above a critical electron density $n_{c}$ and a strong dependence on magnetic fields applied parallel to the plane of the electrons. ${ }^{10}$ A recent theory of Zala, Narozhny, and Aleiner ${ }^{11,12}$ (ZNA) attempts to account for the behavior of the conductivity of these strongly interacting electron systems using a Fermi liquid approach. Within this theory, the temperature and field dependence of the correction to the conductivity due to electron-electron interaction arises from the suppression of quantum interference between different electron trajectories (i.e., coherent scattering off Friedel oscillations).

The ZNA theory describes the behavior of several independently measurable quantities (zero-field conductivity, magnetoconductivity in a parallel field, Hall coefficient, etc.) in terms of a small set of phenomenological parameters. A number of approximations are made in the theory, including using the model of white noise disorder, approximating the Landau function by a single constant, and neglecting intervalley scattering in the case of silicon. We note that although the qualitative features of the temperature dependence should not be sensitive to them, these approximations may significantly alter the results quantitatively. In contrast, these issues will have only a weak effect on the magnetic field dependence, which is associated with the suppression of spinexchange processes. A quantitative comparison with the measured magnetoconductance is therefore a more meaningful test of the applicability of the theory than a comparison with the temperature dependence.

In this paper we present a detailed quantitative comparison of the ZNA theory ${ }^{11,12}$ with the in-plane magnetoconductivity of the 2D electron system in $\mathrm{Si}$ metal-oxidesemiconductor field effect transistors (MOSFET's) measured at different temperatures and different electron densities. Excellent agreement between theory and experiment is obtained for the magnetic field dependence, allowing the determination of the Fermi liquid constant $F_{0}^{\sigma}$ and valley splitting en$\operatorname{ergy} \Delta_{V}$. However, these values of $F_{0}^{\sigma}$ and $\Delta_{V}$ do not pro- vide good fits to the temperature dependence of the zero-field conductivity. We attribute this to the presence of additional scattering processes not included in the theory. ${ }^{11,12}$

We begin with a very brief review of the theory. The conductivity of a disordered electron system (characterized by the large dimensionless conductance $g=2 \pi \hbar / e^{2} R_{\square} \gg 1$; $R_{\square}$ is the sheet resistance) can be expressed as follows: ${ }^{13}$

$$
\sigma=\sigma_{D}+\delta \sigma_{W L}+\delta \sigma_{e e}+\mathcal{O}\left(\frac{1}{g}\right)
$$

Here the Drude conductivity is $\sigma_{D}=n e^{2} \tau / m^{*}, n$ is the electron density, $\tau$ is the elastic mean free time (also see the discussion below), and $m^{*}$ is the effective mass. The weak localization (WL) correction $^{13}$

$$
\delta \sigma_{W L}=-\frac{e^{2}}{2 \pi^{2} \hbar} \ln \frac{\tau_{\varphi}}{\tau}
$$

depends on external parameters through the dephasing time $\tau_{\varphi}$ (see Refs. 13-15 for details). The resulting temperature dependence is logarithmic in zero magnetic field. Based on Eq. (2) and the available data, ${ }^{16}$ one can estimate the value of the WL correction (2). For electron density $n=2.47$ $\times 10^{11} \mathrm{~cm}^{-2}$ used in this work (see below), Eq. (2) yields $\delta \sigma_{W L}=-0.42 \times e^{2} / h$ at $T=0.25 \mathrm{~K}$ and $\delta \sigma_{W L}=-0.22$ $\times e^{2} / h$ at $T=1 \mathrm{~K}$. These values are much smaller than typical variations of the conductivity with in-plane magnetic field $H$ (see Fig. 1). We therefore neglect the WL correction in the present paper.

The correction term due to interactions, $\delta \sigma_{e e},{ }^{13}$ was recently calculated by ZNA (Ref. 11) for the intermediatetemperature regime. Physically, this correction arises due to coherent scattering off Friedel oscillations. For $H=0$ the result of Ref. 11 consists of the charge (singlet) channel contribution

$$
\delta \sigma_{C}=\frac{e^{2}}{\pi \hbar} \frac{T \tau}{\hbar}\left[1-\frac{3}{8} f(T \tau)\right]-\frac{e^{2}}{2 \pi^{2} \hbar} \ln \frac{E_{F}}{T}
$$

and the triplet channel contribution (i.e., the contribution of spin-exchange processes) 


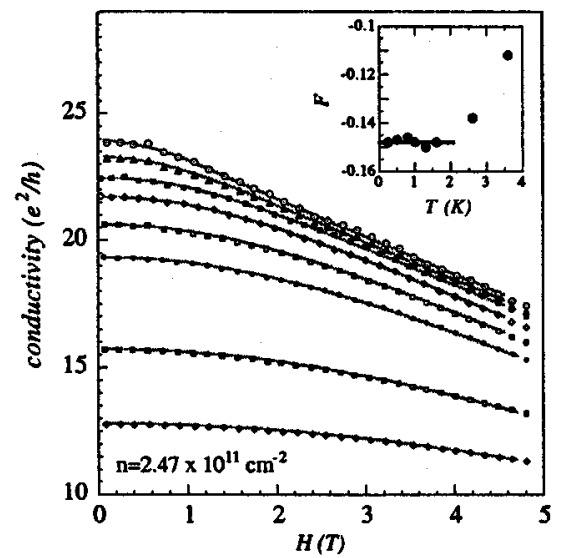

FIG. 1. Conductivity of a Si MOSFET vs in-plane magnetic field at $T=0.25,0.5,0.8,1,1.3,1.6,2.6,3.6$ (K) (from top). Symbols denote the experimental data; solid lines are calculated using Eqs. (3) and (4). The electron density is $n=2.47 \times 10^{11} \mathrm{~cm}^{-2}$. The scattering time used to evaluate Eq. (4) is $\tau=5.28$ ps (see text for discussion). As shown in the inset, the fitting parameter $F_{0}^{\sigma}$ varies above $2 \mathrm{~K}$, indicating that points above this temperature are beyond the range of applicability of the theory.

$$
\begin{aligned}
\delta \sigma_{T}= & \frac{F_{0}^{\sigma}}{\left(1+F_{0}^{\sigma}\right)} \frac{e^{2}}{\pi \hbar} \frac{T \tau}{\hbar}\left[1-\frac{3}{8} t\left(T \tau ; F_{0}^{\sigma}\right)\right] \\
& -\left(1-\frac{1}{F_{0}^{\sigma}} \ln \left(1+F_{0}^{\sigma}\right)\right) \frac{e^{2}}{2 \pi^{2} \hbar} \ln \frac{E_{F}}{T} .
\end{aligned}
$$

Note that the latter must be multiplied by the number of channels ( 3 in the absence of valley degeneracy and the Zeeman splitting $E_{z}=\mu g_{0} H$; the Lande $g$ factor $g_{0}=2$ ):

$$
\delta \sigma_{e e}=\delta \sigma_{C}+3 \delta \sigma_{T} .
$$

The magnetic field suppresses those channels that correspond to nonzero total spin components, giving rise to a dependence of the conductivity on magnetic field: ${ }^{12}$

$$
\begin{aligned}
\delta \sigma\left(E_{z}\right)= & \delta \sigma_{e e}\left(E_{z}\right)-\delta \sigma_{e e}(0) \\
= & \frac{e^{2}}{\pi \hbar}\left[\frac{2 F_{0}^{\sigma}}{\left(1+F_{0}^{\sigma}\right)} \frac{T \tau}{\hbar} \times K_{b}\left(\frac{E_{z}}{2 T}\right)\right. \\
& \left.+K_{d}\left(\frac{E_{z}}{2 \pi T}\right)+m\left(E_{z} \tau, T \tau\right)\right] .
\end{aligned}
$$

Here the ballistic (i.e., dominant for $T \tau \gtrsim 1$ ) contribution to Eq. (3c) is

$$
K_{b}(x)=x \operatorname{coth} x-1+K_{2}\left(x, F_{0}^{\sigma}\right) .
$$

In the above expressions the dimensionless functions $f(x)$, $t\left(x, F_{0}^{\sigma}\right)$, and $m(y, x)$ describe the crossover between the diffusive $(T \tau \ll 1)$ and ballistic regimes. These functions decay smoothly from unity to zero and change the value of $\delta \sigma_{e e}$ by only a few percent outside the crossover region. The details regarding these functions, as well as explicit expressions for
$K_{2}\left(x, F_{0}^{\sigma}\right)$ and the diffusive part of the magnetoconductivity $K_{d}\left(E_{z} / 2 \pi T\right)$, can be found in Refs. 11 and 12 .

The ZNA theory (as described in Refs. 11 and 12 and above) considers an idealized 2D electron gas (2DEG) without taking into account material-dependent details. For 2D electron systems in Si MOSFET's an important additional feature is valley degeneracy. ${ }^{17}$ If we neglect inter-valley scattering (in other words, assume that the valley index is a good quantum number) and assume that the two valleys are degenerate, then we observe that the valley index can be treated as a pseudospin. In this case electrons have not one, but two "spin" indices, and the total number of scattering channels is thus 16 (as opposed to 4 in the usual case considered in Ref. 12). In reality, the valleys are split ${ }^{17}$ with an energy splitting $\Delta_{V}$. The effect of this splitting is similar to the effect of the Zeeman field, since it only shifts energies of the electronic states without affecting the corresponding wave functions.

Taking into account the valley degeneracy, we can write the interaction correction to the conductivity in the following form:

$$
\begin{aligned}
\delta \sigma_{e e}= & \delta \sigma_{C}+15 \delta \sigma_{T}+2 \delta \sigma\left(E_{z}\right)+2 \delta \sigma\left(\Delta_{V}\right)+\delta \sigma\left(E_{z}+\Delta_{V}\right) \\
& +\delta \sigma\left(E_{z}-\Delta_{V}\right),
\end{aligned}
$$

where the last four terms on the right-hand side are defined by Eq. (3c).

Equation (4) is the main theoretical result which we intend to test against the data. Our strategy is the following. The theoretical expressions (3) and (4) contain three phenomenological parameters: the Fermi liquid constant $F_{0}^{\sigma}$, the elastic mean free time $\tau$, and the valley splitting $\Delta_{V}$. As the theory calculates corrections to the impurity scattering time, the value of $\tau$ in all theoretical expressions is the scattering time that the system would have at $T=0$ in the absence of quantum corrections. Therefore $\tau$ must be determined from the temperature dependence of the conductivity: one has to extrapolate the linear temperature dependence from intermediate temperatures to $T=0$ and use the $y$ intercept as the zero-temperature value of the Drude conductivity (thus neglecting the logarithmic corrections; therefore this value does not coincide with the actual measured residual conductivity of the system).

The theory described above is valid ${ }^{11}$ when the dimensionless conductance of the system is large $g \gg 1$ and the system is not too close to the Stoner instability $T<(1$ $\left.+F_{0}^{\sigma}\right)^{2} E_{F}$. Also, the magnetic field dependence, Eq. (4), is valid for relatively weak fields, well below the full polarization of the electron system. The comparison between experiment and theory presented in this paper is restricted to a range of densities and temperatures well within these limits (see below and Figs. 1 and 2).

The remainder of the paper is devoted to a direct comparison of the data with Eq. (4). Measurements were taken on two Si MOSFET's with mobility $\mu \approx 25000 \mathrm{~V} /\left(\mathrm{cm}^{2} \mathrm{~s}\right)$ at $0.3 \mathrm{~K}$. Data were obtained using standard four-terminal ac techniques on samples with split-gate geometry to $12 \mathrm{~T}$ at City College and in fields up to $20 \mathrm{~T}$ at the National High Magnetic Field Laboratory. ${ }^{18}$ The dimensions of the measured portion of the $2 \mathrm{DEG}$ are $120 \times 50 \mu \mathrm{m}^{2}$. Measurements 


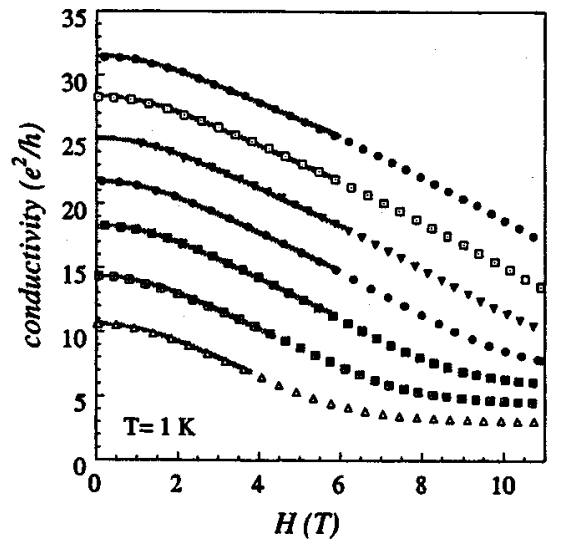

FIG. 2. Conductivity of a Si MOSFET vs in-plane magnetic field for densities $n=3.3,3.0,2.75,2.47,2.19,1.92,1.64$ $\times 10^{11} \mathrm{~cm}^{-2}$ (from top). Symbols denote the experimental data; the lines are calculated using Eq. (4) (for fields weaker than the field for full polarization). The temperature $T=1 \mathrm{~K}$.

were taken at temperatures down to $0.25 \mathrm{~K}$ in the linear regime using small currents of about $1-2 \mathrm{nA}$ to prevent overheating the electrons.

As pointed out earlier, the approximations made in the theory are expected to affect the temperature dependence of the conductivity rather more than the field dependence, which should be relatively robust. We therefore consider a comparison of the magnetoconductivity data with the theory. In Fig. 1 we show the longitudinal conductivity $\sigma_{x x}$ as a function of magnetic field $H_{\|}$applied parallel to the plane of the Si MOSFET for electron density $n=2.47 \times 10^{11} \mathrm{~cm}^{-2}$ at different temperatures. In agreement with earlier results, ${ }^{19}$ there is a substantial decrease of the conductivity with increasing magnetic field. The gray lines are obtained by direct evaluation of Eq. (4) (theoretical results are shifted vertically) using $F_{0}^{\sigma}$ and $\Delta_{V}$ as fitting parameters. The valley splitting only affects the quality of the fit at low fields, while the slope at higher fields depends strongly on the Fermi liquid constant. The determination of the value of $\tau$ used in this calculation is more subtle. In order to extract the value of $\tau$ from the data one needs to know the effective mass of the electrons. One can measure the product $m^{*} g^{*}$ (Ref. 20) experimentally (by analyzing Shubnikov-de Haas data, for instance). The renormalized value of the Lande $g$ factor is related to the same ${ }^{21}$ Fermi liquid constant $g^{*}=g_{0} /(1$ $\left.+F_{0}^{\sigma}\right)$. Using these relations self-consistently, we obtain the value of $\tau$ for each electron density. For the density $n$ $=2.47 \times 10^{11} \mathrm{~cm}^{-2}$ we find $\tau=5.28$ ps.

Excellent agreement between experiment and theory is obtained for the magnetoconductance, as demonstrated in Fig. 1. The fits are relatively insensitive to the value of $\Delta_{V}$, with $\Delta_{V} \approx 1 \mathrm{~K}$ for all electron densities. As shown in the inset to Fig. 1 for density $n_{s}=2.47 \times 10^{11} \mathrm{~cm}^{-2}, F_{0}^{\sigma}$ is essentially constant below $2 \mathrm{~K}$ as required by theory and increases above this temperature; similar behavior was found at other densities. We suggest that this sets an experimentally determined limit on the temperature below which the ZNA theory is applicable. The Fermi liquid parameter $F_{0}^{\sigma}$ is found to be roughly independent of electron density within the

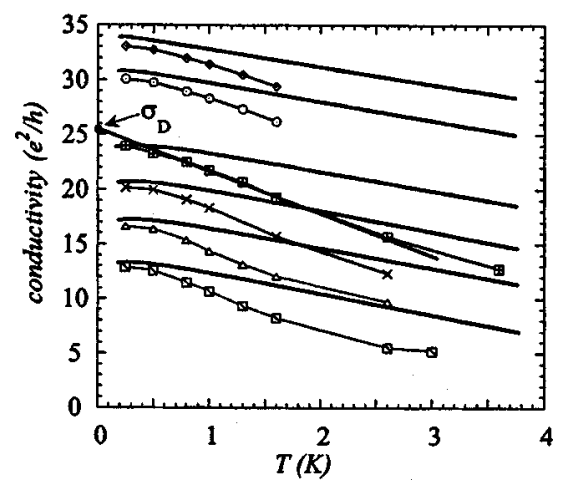

FIG. 3. Temperature dependence of the conductivity of $2 \mathrm{D}$ electrons in a Si MOSFET at $H=0$ for densities $n=3.3,3.0,2.47,2.19$, $1.92,1.64 \times 10^{11} \mathrm{~cm}^{-2}$ (from top). The symbols indicate experimental data. The solid lines are calculated using the theory (Refs. 11 and 12). The straight line drawn for density $2.47 \times 10^{11} \mathrm{~cm}^{-2}$ is an extrapolation to zero temperature of the (nearly) linear part of the conductivity used to determine the value of $\tau=5.28 \mathrm{ps}$ (see text).

range of our experiments: for densities $n=1.6 \times 10^{11} \mathrm{~cm}^{-2}$ and higher, $F_{0}^{\sigma}=-(0.15 \pm 0.01)$.

Figure 2 shows the in-plane magnetoconductivity for different densities at a fixed temperature $T=1 \mathrm{~K}$. The zerofield conductivity is a linear function of temperature in the vicinity of $1 \mathrm{~K}$ (see Fig. 3) and is thus in the ballistic regime $T \tau>0.1$ of the theory. ${ }^{11,12}$ The comparison is made for magnetic field below $6 \mathrm{~T}$, where variations of the magnetoconductivity are relatively small (well within the range of applicability of the calculations). Again, quantitative agreement is obtained between theory and experiment.

We now compare the measured temperature dependence of the conductivity in zero magnetic field with that predicted by the theory ${ }^{11,12}$ using the values of $F_{0}^{\sigma}$ and $\Delta_{V}$ determined above from fits to the field dependence. Figure 3 shows the data (symbols) and the predictions of the theory (solid lines). As noted above and illustrated in the inset to Fig. 1, the theory is not expected to apply for $T>2 \mathrm{~K}$. It is clear, however, that no agreement is obtained even for $T<2 \mathrm{~K}$ : the temperature dependence observed experimentally is considerably stronger than that calculated from the theory. ${ }^{11,12}$

The theoretical prediction shown in Fig. 3 depends on the value used for the electron mass. If we abandon the selfconsistent procedure for determining the renormalized mass $m^{*}$ (see above) and use instead the bare band mass (similar to Ref. 7), the discrepancy between theory and experiment is reduced. However, the slopes of the theoretical and experimental curves still differ by about $30 \%$.

Note that various authors have used the ZNA theory to determine $F_{0}^{\sigma}$ and $\Delta_{V}$ by fitting to the temperature dependence of $\delta \sigma_{e e}$ rather than the field dependence (see also Ref. 22). Our attempts to do this yield values of $F_{0}^{\sigma}$ that are much larger than those deduced from the field dependence above and similar to those obtained in Ref. 23. In addition to the fact that the temperature dependence is more sensitive to the approximations in the theory as argued above, this approach is also less reliable for our data: the temperature dependence 
in Fig. 3 exhibits linear behavior over a very narrow range, unlike the magnetoconductivity which exhibits a broad range of linear dependence on field (see Fig. 1). This is consistent with the theoretical expectations discussed above.

In summary, for the strongly interacting two-dimensional system of electrons in silicon MOSFET's, excellent agreement between experiment and the theory of Zala, Narozhny, and Aleiner ${ }^{11,12}$ was obtained for the response of the conductivity to a magnetic field applied parallel to the plane of the electrons for $T<2 \mathrm{~K}$. For temperatures between $0.25 \mathrm{~K}$ and $2 \mathrm{~K}$ and densities down to $1.64 \times 10^{11} \mathrm{~cm}^{-2}$, the theory provides quantitative agreement with the measured magnetic field dependence of the conductivity using only two parameters: the valley splitting $\Delta=1 \mathrm{~K}$ and the Fermi liquid parameter $F_{0}^{\sigma} \approx-0.15$. Although the parameters $F_{0}^{\sigma}(n)$ and $\Delta_{V}$ obtained from fits to the magnetoconductivity provide qualitatively correct behavior for the temperature dependence of the conductivity in zero magnetic field (including the sign), they do not yield quantitative agreement. While including additional scattering processes, such as intervalley scattering, could result in better agreement, it would be at the price of introducing additional fitting parameters, thus making the fits less meaningful.

The results reported in this paper indicate that the ZNA theory provides a good description of the behavior of $2 \mathrm{D}$ electron systems in Si MOSFETs for densities above 1.64 $\times 10^{-11} \mathrm{~cm}^{-2}$. At lower densities the system exhibits much larger variations of observed conductivity, particularly as a function of in-plane mangetic field. The behavior at very low densities remains poorly understood and requires further investigation.

We are grateful to I. L. Aleiner, D. L. Maslov, and B. Spivak for helpful discussions. This work was supported by the U.S. Department of Energy at City College under Grant No. DE-FG02-84ER45153 and at Brookhaven under Contract No. DE-AC02-98 CH 10886.
${ }^{1}$ S. V. Kravchenko, G. V. Kravchenko, J. E. Furneaux, V. M. Pudalov, and M. D'Iorio, Phys. Rev. B 50, 8039 (1994); S. V. Kravchenko, D. Simonian, M. P. Sarachik, Whitney Mason, and J. E. Furneaux, Phys. Rev. Lett. 77, 4938 (1996).

${ }^{2}$ S. J. Papadakis and M. Shayegan, Phys. Rev. B 57, R15 068 (1998).

${ }^{3}$ Y. Hanein, D. Shahar, J. Yoon, C. C. Li, D. C. Tsui, and H. Shtrikman, Phys. Rev. B 58, R13 338 (1998).

${ }^{4}$ P. T. Coleridge, R. L. Williams, Y. Feng, and P. Zawadzki, Phys. Rev. B 56, R12 764 (1997).

${ }^{5}$ Y. Hanein, U. Meirav, D. Shahar, C. C. Li, D. C. Tsui, and H. Shtrikman, Phys. Rev. Lett. 80, 1288 (1998).

${ }^{6}$ M. Y. Simmons, A. R. Hamilton, M. Pepper, E. H. Linfield, P. D. Rose, D. A. Ritchie, A. K. Savchenko, and T. G. Griffiths, Phys. Rev. Lett. 80, 1292 (1998).

${ }^{7}$ Y. Y. Proskuryakov, A. K. Savchenko, S. S. Safonov, M. Pepper, M. Y. Simmons, and D. A. Ritchie, Phys. Rev. Lett. 89, 076406 (2002).

${ }^{8}$ P. T. Coleridge, A. S. Sachrajda, and P. Zawadzki, Phys. Rev. B 65, 125328 (2002)

${ }^{9}$ E. Abrahams, S. V. Kravchenko, and M. P. Sarachik, Rev. Mod. Phys. 73, 251 (2001).

${ }^{10}$ S. A. Vitkalov, H. Zheng, K. M. Mertes, M. P. Sarachik, and T. M. Klapwijk, Phys. Rev. Lett. 87, 086401 (2001).

${ }^{11}$ Gabor Zala, B. N. Narozhny, and I. L. Aleiner, Phys. Rev. B 64, 214204 (2001).

${ }^{12}$ Gabor Zala, B. N. Narozhny, and I. L. Aleiner, Phys. Rev. B 65, 020201 (2001).

${ }^{13}$ B. L. Altshuler and A. G. Aronov, in Electron-Electron Interac- tions in Disordered Systems, edited by A. L. Efros and M. Pollak (North-Holland, Amsterdam, 1985).

${ }^{14}$ I. L. Aleiner, B. L. Altshuler, and M. E. Gershenson, Waves Random Media 9, 201 (1999).

${ }^{15}$ B. N. Narozhny, Gabor Zala, and I. L. Aleiner, cond-mat/0201379 (unpublished).

${ }^{16}$ G. Brunthaler, A. Prinz, G. Bauer, and V. M. Pudalov, Phys. Rev. Lett. 87, 096802 (2001).

${ }^{17}$ T. Ando, A. B. Fowler, and F. Stern, Rev. Mod. Phys. 54, 437 (1982).

${ }^{18}$ S. A. Vitkalov, H. Zheng, K. M. Mertes, M. P. Sarachik, and T. M. Klapwijk, Phys. Rev. Lett. 85, 2164 (2000).

${ }^{19}$ D. Simonian, S. V. Kravchenko, M. P. Sarachik, and V. M. Pudalov, Phys. Rev. Lett. 79, 2304 (1997).

${ }^{20}$ S. A. Vitkalov, M. P. Sarachik, and T. M. Klapwijk, Phys. Rev. B 65, 201106 (2002).

${ }^{21}$ The constant that describes the susceptibility is the zeroth harmonic of the spin part of the Landau function $f(\theta)$, while the constant that describes the conductivity in the ballistic regime corresponds to backscattering, i.e., $\theta=\pi$. The ZNA theory (Ref. 11) assumes that the Fermi liquid parameter is independent of $\theta$ and thus uses just one constant $F_{0}^{\sigma}$ to provide a phenomenological description of interaction in the triplet channel.

${ }^{22}$ V. M. Pudalov, M. Gershenson, H. Kojima, N. Busch, E. M. Dizhur, G. Brunthaler, A. Prinz, and G. Bauer, cond-mat/0206258 (unpublished).

${ }^{23}$ A. A. Shashkin, S. V. Kravchenko, V. T. Dolgopolov, and T. M. Klapwijk, Phys. Rev. B 66, 073303 (2002). 